\title{
Testes Psicológicos em Portugal: Atitudes, Problemas e Perfil dos Utilizadores
}

\author{
Psychological Tests in Portugal: Attitudes, Problems and User Profiles
}

\author{
Maria João Seabra-Santos ${ }^{1}$, Pedro Armelim Almiro ${ }^{2}$, Mário R. Simões ${ }^{3}$ e Leandro S. Almeida ${ }^{4}$
}

\begin{abstract}
Resumo
Este artigo pretende caracterizar aspetos relevantes da situação atual do uso de testes psicológicos em Portugal. A amostra foi composta por 1523 psicólogos que preencheram um questionário online sobre práticas, atitudes e problemas identificados no uso de testes. Os resultados mostram que a grande maioria dos respondentes (cerca de 90\%) usa testes na sua prática profissional, fazendo-o, maioritariamente, com objetivo de avaliação/diagnóstico ou de rastreio, e para avaliar sujeitos em idade escolar ou adultos até aos 64 anos. Os resultados sublinham: uma atitude favorável relativamente à importância da formação sobre testes, considerando que estes podem ser de grande ajuda; a necessidade de legislação e normas nesta área; uma adesão clara à ideia de que o uso de testes deve ser uma atividade da exclusiva responsabilidade dos psicólogos. O fazer fotocópias dos testes e o não contrastar os resultados obtidos com outros colegas foram alguns dos problemas identificados.
\end{abstract}

Palavras-chave: testes psicológicos, avaliação psicológica, perfil do utilizador, atitudes

\begin{abstract}
This article aims to describe relevant aspects of the current status of the use of psychological tests in Portugal. The sample consisted of 1523 psychology professionals and was based on their answers to an online questionnaire about practices, attitudes and problems related to psychological testing. The results show that a vast majority of respondents (aprox. 90\%) use tests in their professional practice, mostly for evaluation/diagnosis or screening purposes, and to evaluate subjects from school age up to 64 years of age. The results underline a favorable attitude towards the importance of training on testing methods, and the consideration that these can be of great help, the need for legislation and standards in this specific area, and a clear adherence to the idea that tests should be used solely by psychologists. Making photocopies of tests, and not cross-checking the results obtained with other colleagues were some of the problems identified.
\end{abstract}

Keywords: psychological tests, psychological assessment, user profiles, attitudes

Agradecimentos: Os autores estão gratos à Ordem dos Psicólogos Portugueses (OPP) pelo envio aos psicólogos portugueses de um convite à sua participação no estudo.

\footnotetext{
${ }^{1}$ Doutora em Psicologia. Professora Auxiliar na Universidade de Coimbra. Faculdade de Psicologia e de Ciências da Educação da Universidade de Coimbra, CINEICC - Centro de Investigação em Neuropsicologia e Intervenção Cognitivo-Comportamental, PsyAssessmentLab. Rua do Colégio Novo, 3000-115 Coimbra, Portugal. Tel.: 00351 239851450. E-mail: seabramj@ fpce.uc.pt

${ }^{2}$ Doutor em Psicologia. Professor Auxiliar na Universidade Autónoma de Lisboa. Departamento de Psicologia da Universidade Autónoma de Lisboa, CIP - Centro de Investigação em Psicologia, CINEICC - Centro de Investigação em Neuropsicologia e Intervenção Cognitivo-Comportamental, PsyAssessmentLab. Rua de Santa Marta, 47, 1150-293 Lisboa, Portugal. Tel.: 00351 213177600. E-mail: paalmiro@autonoma.pt

${ }^{3}$ Doutor em Psicologia. Professor Catedrático na Universidade de Coimbra. Faculdade de Psicologia e de Ciências da Educação da Universidade de Coimbra, CINEICC - Centro de Investigação em Neuropsicologia e Intervenção Cognitivo-Comportamental, PsyAssessmentLab. Rua do Colégio Novo, 3000-115 Coimbra, Portugal. Tel.: 00351 239851450. E-mail: simoesmr@ fpce.uc.pt

${ }^{4}$ Doutor em Psicologia. Professor Catedrático na Universidade do Minho. Instituto de Educação da Universidade do Minho, CIED Centro de Investigação em Educação. Campus de Gualtar, 4710-057 Braga, Portugal. Tel.: 00351 253604255. E-mail: leandro@ie.uminho.pt

Revista Iberoamericana de Diagnóstico y Evaluación - e Avaliação Psicológica. RIDEP · No53 · Vol.4 · 101-112 · 2019

ISSN: 1135-3848 print /2183-6051online
} 


\section{Introdução}

A avaliação psicológica integra, ao longo dos tempos, uma lista reduzida de práticas confinadas ou identitárias da profissão de psicólogo. Neste sentido, explica-se ser objeto de investigação nas formas que assume e nas perceções das suas potencialidades e dificuldades junto dos psicólogos, sendo esta análise enriquecida pela confrontação de resultados entre países. Esta análise permite várias inferências sobre a formação e o exercício da psicologia, objetivos que vão para além da própria caraterização da avaliação psicológica em contextos nacionais e internacionais.

Em consonância, organismos internacionais e nacionais representativos da ciência e profissão psicológica realizam, de forma periódica, levantamentos sobre a prática da avaliação psicológica, em particular acerca do uso dos testes e outros instrumentos, com o objetivo de identificar lacunas e monitorizar desenvolvimentos na área. Uma atenção particular é dada ao tipo de testes e outros instrumentos de avaliação utilizados (e.g., escalas, questionários, inventários), mas igualmente se analisam as práticas instituídas e as atitudes inerentes (Archer, Buffington-Vollum, Stredny, \& Handel, 2006; Bornstein, 2017; Camara, Nathan, \& Puente, 2000). Dada a centralidade da avaliação psicológica na prática da psicologia, estes estudos permitem análises evolutivas sobre a formação inicial e contínua dos psicólogos, os instrumentos e versões disponíveis, e as opções dos psicólogos na escolha dos testes e instrumentos que utilizam.

Numa breve resenha de estudos que conhecemos em Portugal sobre este tópico, um primeiro estudo analisou a utilização dos testes psicológicos junto de 110 psicólogos, numa época em que a prática da Psicologia em Portugal estava ainda a dar os primeiros passos (Almeida \& Cruz, 1985; Cruz \& Almeida, 1987). Os resultados mostraram que estes profissionais tinham uma atitude de aceitação face ao método dos testes e percecionavam também, por parte do público em geral e de outros profissionais com quem partilhavam os seus contextos de trabalho, uma postura de aceitação do uso de testes. Os inquiridos identificaram como principais problemas dos testes a não existência de revisões periódicas e o facto de não atenderem aos processos psicológicos subjacentes, apontando para a necessidade das associações científicas e profissionais de Psicologia fixarem e zelarem pelo cumprimento de princípios regulamentadores do uso de testes psicológicos.

Um segundo inquérito foi realizado uma década e meia mais tarde, junto de 41 especialistas em avaliação psicológica oriundos de 21 países Ibero-Americanos, incluindo Portugal (Muñiz, Prieto, Almeida, \& Bartram, 1999). Neste estudo foram identificados problemas associados ao uso dos testes nos países participantes, alguns deles idênticos aos encontrados noutros países não abrangidos pelo inquérito, tais como o uso de fotocópias, o uso de testes inapropriados ou desatualizados, a utilização dos resultados sem ter em conta o erro de medida ou o uso por profissionais não qualificados para tal utilização. Outras dificuldades identificadas mostravam-se mais específicas destes países, decorrentes do facto de muitos dos testes utilizados serem importados de outras culturas, apresentando traduções e adaptações inadequadas e não ponderando suficientemente condições que podem afetar a validade do teste num país diferente. Também neste estudo foi sinalizada a importância de as associações profissionais assumirem maior responsabilidade na regulação do uso dos testes.

Cerca de uma década mais tarde, foi conduzido um terceiro inquérito sobre práticas de avaliação psicológica, junto de 265 psicólogos portugueses (Diniz, Almeida, \& Pais, 2007). Neste estudo, a realização de tarefas de avaliação psicológica surgiu como prática bastante generalizada pelos psicólogos respondentes, atravessando os vários contextos da sua atuação profissional. Em defesa desta utilização generalizada, os respondentes assumiam a avaliação psicológica como relevante para se assegurar maior precisão e rigor à prática psicológica, constituindo também uma marca identitária da mesma. Embora a maioria dos inquiridos considerasse adequada a formação recebida nesta área, identificavam a formação e supervisão pós-graduada como necessárias para uma mais correta utilização das provas psicológicas em Portugal. Uma outra preocupação dos participantes residia na necessidade de validação de provas para Portugal, em 
convergência com o facto de muitas das provas elencadas como sendo utilizadas pelos respondentes não se encontrarem validadas para a população portuguesa.

Face ao exposto, e no quadro de um estudo internacional envolvendo vários países iberoamericanos, este artigo centra-se nos dados recolhidos em Portugal, pretendendo caracterizar aspetos considerados relevantes quanto à situação atual do uso de testes e outros instrumentos de avaliação psicológica. Mais especificamente, é feita uma análise comparativa do perfil dos psicólogos que usam e dos que não usam instrumentos de avaliação psicológica na sua prática e são elencadas as razões invocadas para o não-uso. Para os psicólogos que usam testes e outros instrumentos de avaliação são identificadas as respetivas áreas profissionais e áreas de especialização, assim como os objetivos da utilização (e.g., rastreio, diagnóstico) e idades da população destinatária. Finalmente, são analisados problemas e atitudes associados à utilização de testes psicológicos.

\section{Método}

\section{Amostra}

Um total de 1921 psicólogos portugueses acederam ao site após convite da Ordem dos Psicólogos Portugueses (OPP) para colaborarem no estudo, contudo alguns não iniciaram $\mathrm{o}$ preenchimento $(n=211 ; 11.0 \%)$ e outros não foram além da caraterização inicial do respondente $(n=187 ; 9.7 \%)$. As análises reportadas neste artigo incidem sobre os restantes 1523 respondentes. Estes são maioritariamente do sexo feminino $(87.2 \%)$, registando-se apenas 195 respondentes do sexo masculino (12.8\%). A maioria possui como grau académico mais elevado o mestrado ( $n=740 ; 49.0 \%)$, havendo 408 respondentes $(27.0 \%)$ com a licenciatura (formação pré-Bolonha), assim como $227 \mathrm{com}$ curso de especialização pós-licenciatura (15.0\%) e 136 com doutoramento $(9.0 \%)$. No sentido de encontrar uma formulação comum, adequada aos vários países participantes da pesquisa, a sequência colocada no questionário quanto ao nível de formação foi: licenciatura/graduação, especialização, mestrado e doutoramento. Tal não nos permite afirmar se a especialização apontada pelos respondentes portugueses significa uma qualificação académica/profissional posterior à licenciatura ou ao mestrado. Porém, dada a sequência colocada na questão, este tipo de resposta terá tendencialmente a primeira interpretação. Aliás, nesta questão registaram-se 12 casos omissos que poderão estar relacionados com alguma dificuldade sentida na sua resposta.

As idades dos respondentes oscilam entre $24 \mathrm{e}$ 76 anos $(M d=38.00 ; M=39.57, D P=9.25)$, com maior concentração na faixa dos 30 aos 40 anos (até 30 anos situa-se $15.7 \%$ da amostra, e com 60 ou mais anos regista-se $3.1 \%$ dos respondentes). A idade dos respondentes está associada com o número de anos de prática profissional como psicólogos $(r=.84, p<.001)$, registando-se, nesta última variável, um leque de valores entre um $(n=32)$ e 45 anos $(n=1)$. A moda situa-se nos 10 anos $(n=123)(M d=12.00, M=13.32, D P=8.35)$, havendo uma maior concentração de valores entre os 10 e os 15 anos e registando-se quatro casos omissos.

\section{Instrumento}

Os coordenadores do estudo em cada um dos países acordaram num questionário comum procurando, na formulação dos itens, atender a especificidades da formação e exercício profissional da Psicologia em cada país, no sentido de aumentar a objetividade das respostas e facilitar futuras comparações dos dados obtidos. O questionário aplicado incluía várias secções: (i) Breve caraterização do(a) psicólogo(a) respondente, nomeadamente Idade, Sexo, País, Região, Nível de formação, Campo de atuação atual, Experiência profissional (anos), Áreas de formação na pós-graduação, Área de prática na Psicologia; (ii) Descrição da utilização de testes psicológicos, nomeadamente Recurso habitual a testes psicológicos; Razões se não recorre; Objetivo (Diagnóstico, Recrutamento, Orientação ou aconselhamento, Avaliação de programas); População (idades) e Testes usados na prática profissional; (iii) Atitudes face à formação e utilização de provas psicológicas (por exemplo, $A$ formação recebida no curso é suficiente para a prática profissional; Utilizados corretamente, os testes são grande ajuda); à necessidade de regulamentação no seu uso (por exemplo, $E$ 
necessária legislação oficial específica para controlar o uso dos testes); e ao uso exclusivo destes instrumentos por parte de psicólogos (por exemplo, $O$ uso dos testes deve ser limitado a psicólogos/as qualificados/as); e (iv) Problemas identificados, por exemplo Fazer fotocópias de testes, Utilização de testes inadequados, Insuficiente atualização nos testes que usa, Não considerar variáveis regionais que podem afetar a validade dos testes. $\mathrm{O}$ questionário privilegia um formato de questões fechadas, para mais fácil análise das respostas, havendo, contudo, algumas questões abertas, nomeadamente quanto às razões de não uso de testes, objetivos da sua utilização e identificação dos testes mais utilizados. $\mathrm{O}$ instrumento termina com uma questão aberta, que possibilita aos respondentes acrescentar algum comentário relativo ao uso de testes no seu país.

\section{Procedimentos}

O questionário foi aplicado online sendo as respostas diferenciadas pelo país dos psicólogos respondentes. No início apresentavam-se os objetivos do estudo e a relevância do maior número de resposta para assegurar maior robustez nas análises e conclusões, mesmo para legitimar o objetivo de comparação de respostas entre países. Para além de não ser requerida a identificação pessoal, assegurou-se o tratamento anónimo dos resultados.

As análises estatísticas dos dados foram realizadas recorrendo ao programa IBM SPSS Statistics 24 (IBM Corp., Armonk, NY). Foram calculados indicadores de estatística descritiva (frequências, percentagens, moda, mediana, média e desvio-padrão) para caracterizar a amostra do ponto de vista demográfico e quanto à área de atividade profissional e de especialização. O coeficiente de correlação de Pearson permitiu analisar a associação entre a idade dos respondentes e o número de anos de prática profissional como psicólogos. Utilizaram-se os testes $\chi^{2}$ e $t$ de Student para comparar as características demográficas dos respondentes que usam instrumentos de avaliação psicológica com os que não usam. Este último teste foi também usado para comparar as respostas dos dois tipos de respondentes quanto a atitudes e problemas face à utilização de instrumentos de avaliação psicológica. Recorreu-se à análise ANOVA, seguida do teste de Bonferroni para analisar contrastes, para comparar as respostas de participantes de diferentes áreas de atividade profissional quanto ao objetivo do uso de instrumentos de avaliação e à faixa etária da população-alvo.

\section{Resultados}

Antes de prosseguirmos na apresentação dos resultados importa referir que à questão "Na sua atividade profissional utiliza habitualmente testes e/ou questionários psicológicos?", 140 respondentes referiram "Não" $(9.2 \%)$, o que justifica a sua análise diferenciada, pois não são considerados na descrição posterior das práticas de avaliação. As razões apontadas para o não uso (considerando os que justificaram essa opção de resposta): 28 respondentes justificam que a sua área de intervenção (ação social, investigação, docência, direção/coordenação de serviços de psicologia, prática clínica, psicoterapia) não torna necessária a realização da avaliação psicológica e em particular o uso de testes e outros instrumentos; 10 respondentes apontam que a avaliação psicológica não se adequa e não é aplicável, sem outras justificações; três respondentes apontam que a instituição onde trabalham não adquiriu testes; dois referem a falta de instrumentos para as populações específicas de trabalho; dois apontam a falta de tempo e condições; dois mencionam a sua preferência pelo recurso exclusivo à entrevista; por último, registase um respondente para cada um dos seguintes argumentos: estar desempregado, a avaliação não estar prevista no planeamento das atividades, não ver benefício no recurso à avaliação psicológica nas situações habituais da sua prática profissional e não realização de avaliação formal.

Analisando as subamostras de respondentes que usam e que não usam habitualmente as provas psicológicas, regista-se uma taxa ligeiramente superior de psicólogas que usam ou de psicólogos que não usam $\left(\chi^{2}=5.80, g l=1, p<.05\right)$, não se registando uma diferença estatisticamente significativa entre a utilização ou não em função do nível da formação máxima possuída $\left(\chi^{2}=1.49\right.$, $g l=3, p=.684)$. No grupo de psicólogos que usa testes regista-se uma média de idades superior 
Quadro 1. Atitudes face à formação e utilização de provas psicológicas

\begin{tabular}{|c|c|c|c|c|c|}
\hline & \multicolumn{2}{|c|}{ Amostra total } & \multirow{2}{*}{$\begin{array}{c}\text { Utilizadores } \\
M \\
(D P)\end{array}$} & \multirow{2}{*}{$\begin{array}{c}\text { Não-utilizadores } \\
M \\
(D P)\end{array}$} & \multirow[b]{2}{*}{$t$} \\
\hline & Mo & $\begin{array}{c}M \\
(D P)\end{array}$ & & & \\
\hline Controlo mínimo pois inibe o desenvolvimento & 2 & $\begin{array}{c}2.28 \\
(1.02)\end{array}$ & $\begin{array}{c}2.28 \\
(1.02)\end{array}$ & $\begin{array}{c}2.22 \\
(1.03)\end{array}$ & -.49 \\
\hline $\begin{array}{l}\text { Curso disciplinas de construção e avaliação de } \\
\text { testes }\end{array}$ & 5 & $\begin{array}{l}4.25 \\
(.84)\end{array}$ & $\begin{array}{l}4.26 \\
(.83)\end{array}$ & $\begin{array}{l}4.13 \\
(.89)\end{array}$ & -1.21 \\
\hline Editores podem comercializar qualquer teste ${ }^{1}$ & 1 & $\begin{array}{c}2.39 \\
(1.22)\end{array}$ & $\begin{array}{c}2.39 \\
(1.22)\end{array}$ & $\begin{array}{c}2.33 \\
(1.20)\end{array}$ & -.40 \\
\hline Satisfeito(a) com a qualidade dos testes que utilizo & 4 & $\begin{array}{l}3.54 \\
(.89)\end{array}$ & $\begin{array}{l}3.57 \\
(.89)\end{array}$ & $\begin{array}{l}3.14 \\
(.86)\end{array}$ & $-3.86^{* *}$ \\
\hline Na minha prática, o uso de testes dá-me confiança & 4 & $\begin{array}{l}3.83 \\
(.90)\end{array}$ & $\begin{array}{l}3.87 \\
(.88)\end{array}$ & $\begin{array}{l}3.28 \\
(.94)\end{array}$ & $-5.34 * *$ \\
\hline A internet pode melhorar administração dos testes & 3 & $\begin{array}{c}3.27 \\
(1.06)\end{array}$ & $\begin{array}{c}3.27 \\
(1.05)\end{array}$ & $\begin{array}{c}3.20 \\
(1.11)\end{array}$ & -.51 \\
\hline Úteis se complementados com outras informações & 5 & $\begin{array}{l}4.60 \\
(.75)\end{array}$ & $\begin{array}{l}4.60 \\
(.75)\end{array}$ & $\begin{array}{l}4.64 \\
(.71)\end{array}$ & .45 \\
\hline $\begin{array}{l}\text { Utilizados corretamente, os testes são de grande } \\
\text { ajuda }\end{array}$ & 5 & $\begin{array}{l}4.58 \\
(.69)\end{array}$ & $\begin{array}{l}4.60 \\
(.68)\end{array}$ & $\begin{array}{l}4.26 \\
(.74)\end{array}$ & $-3.95 * *$ \\
\hline Formação no curso é suficiente & 3 & $\begin{array}{c}2.67 \\
(1.11)\end{array}$ & $\begin{array}{c}2.66 \\
(1.11)\end{array}$ & $\begin{array}{l}2.75 \\
(1.10)\end{array}$ & .69 \\
\hline $\begin{array}{l}\text { Conhecimentos foram adquiridos no curso de } \\
\text { Psicologia }\end{array}$ & 2 & $\begin{array}{c}2.48 \\
(1.07)\end{array}$ & $\begin{array}{c}2.46 \\
(1.06)\end{array}$ & $\begin{array}{c}2.71 \\
(1.24)\end{array}$ & 1.64 \\
\hline $\begin{array}{l}\text { Profissionais têm suficiente informação sobre } \\
\text { qualidade dos testes }\end{array}$ & 3 & $\begin{array}{c}2.56 \\
(1.00)\end{array}$ & $\begin{array}{c}2.56 \\
(1.00)\end{array}$ & $\begin{array}{l}2.49 \\
(.96)\end{array}$ & -.56 \\
\hline
\end{tabular}

Questão formulada em sentido inverso ao desejável.

Quadro 2. Atitudes face à regulamentação do uso de provas psicológicas

\begin{tabular}{|c|c|c|c|c|c|}
\hline & \multicolumn{2}{|c|}{ Amostra total } & \multirow{2}{*}{$\begin{array}{c}\text { Utilizadores } \\
M \\
(D P)\end{array}$} & \multirow{2}{*}{$\begin{array}{c}\text { Não-utilizadores } \\
M \\
(D P)\end{array}$} & \multirow[b]{2}{*}{$t$} \\
\hline & Mo & $\begin{array}{c}M \\
(D P)\end{array}$ & & & \\
\hline $\begin{array}{l}\text { Associações profissionais mais ativas na } \\
\text { regulação }\end{array}$ & 4 & $\begin{array}{l}3.95 \\
(.93)\end{array}$ & $\begin{array}{l}3.94 \\
(.94)\end{array}$ & $\begin{array}{l}4.06 \\
(.86)\end{array}$ & 1.00 \\
\hline Certificar a competência dos utilizadores de testes & 4 & $\begin{array}{c}3.70 \\
(1.14)\end{array}$ & $\begin{array}{c}3.68 \\
(1.14)\end{array}$ & $\begin{array}{c}3.90 \\
(1.05)\end{array}$ & 1.51 \\
\hline Legislação para controlar uso de testes & 5 & $\begin{array}{c}4.12 \\
(1.03)\end{array}$ & $\begin{array}{c}4.12 \\
(1.03)\end{array}$ & $\begin{array}{l}4.06 \\
(1.01)\end{array}$ & -.50 \\
\hline Normas obrigatórias sobre qualidade dos testes & 4 & $\begin{array}{l}3.93 \\
(.93)\end{array}$ & $\begin{array}{l}3.93 \\
(.94)\end{array}$ & $\begin{array}{l}3.87 \\
(.89)\end{array}$ & -.54 \\
\hline Mais controlo por organismos profissionais & 5 & $\begin{array}{c}3.94 \\
(1.07)\end{array}$ & $\begin{array}{c}3.94 \\
(1.08)\end{array}$ & $\begin{array}{l}3.93 \\
(.98)\end{array}$ & -.08 \\
\hline
\end{tabular}

$(t=2.58, g l=1520, p<.05)$ e também um maior número de anos de prática $(t=2.64, g l=1517$, $p<.01)$. Considerando a área de prática, regista-se maior proporção de respostas "não uso" na área Social-Comunitária (31.5\%) e mais respostas "uso" em Neuropsicologia, Psicologia Escolar e da Educação e Psicologia Clínica e da Saúde (sempre acima de 90\%) $\left(\chi^{2}=123.19, g l=8, p<.001\right)$.

\section{Atitudes face à utilização de provas psicológicas e problemas identificados}

Os Quadros 1 a 4 apresentam os resultados relativos às questões sobre atitudes face à utilização de instrumentos de avaliação psicológica e problemas identificados. Embora, nestas análises, se tenha tido em consideração a totalidade da amostra (quer os psicólogos que utilizam, quer os que não utilizam estes instrumentos), o número de respostas para cada item oscila entre 1029 e 1032, havendo uma percentagem de cerca de $32 \%$ da amostra que não respondeu a este conjunto de itens. As respostas foram dadas numa escala likert de 5 pontos, desde "totalmente em desacordo" (1) a "totalmente de acordo" (5).

No que diz respeito às atitudes face à formação e utilização de provas psicológicas (Quadro 1), a amostra deste estudo destaca a importância da existência de unidades curriculares nos cursos de Psicologia sobre construção e avaliação de testes, considerando que estes são de grande ajuda quando complementados com outras informações e usados corretamente e, ainda, discordando que os editores devam ter autorização para comercializar qualquer teste. As atitudes dos utilizadores diferem de forma estatisticamente significativa das posições dos não utilizadores 
Quadro 3. Atitudes sobre a exclusividade da utilização de testes por psicólogos

\begin{tabular}{|c|c|c|c|c|c|}
\hline & \multicolumn{2}{|c|}{ Amostra total } & \multirow{2}{*}{$\begin{array}{c}\text { Utilizadores } \\
M \\
(D P)\end{array}$} & \multirow{2}{*}{$\begin{array}{c}\text { Não-utilizadores } \\
M \\
(D P)\end{array}$} & \multirow[b]{2}{*}{$t$} \\
\hline & Mo & $\begin{array}{c}M \\
(D P)\end{array}$ & & & \\
\hline $\begin{array}{l}\text { Interpretação dos resultados só por } \\
\text { psicólogos/as }\end{array}$ & 5 & $\begin{array}{l}4.77 \\
(.71)\end{array}$ & $\begin{array}{l}4.77 \\
(.71)\end{array}$ & $\begin{array}{l}4.72 \\
(.71)\end{array}$ & -.55 \\
\hline Resultados comunicados só por psicólogos/as & 5 & $\begin{array}{l}4.63 \\
(.79)\end{array}$ & $\begin{array}{l}4.63 \\
(.79)\end{array}$ & $\begin{array}{l}4.58 \\
(.79)\end{array}$ & -.50 \\
\hline $\begin{array}{l}\text { Testes administrados e cotados por não } \\
\text { psicólogos/as }{ }^{1}\end{array}$ & 1 & $\begin{array}{l}1.44 \\
(.96)\end{array}$ & $\begin{array}{l}1.44 \\
(.96)\end{array}$ & $\begin{array}{l}1.45 \\
(.92)\end{array}$ & .10 \\
\hline Uso limitado a psicólogos/as qualificados/as & 5 & $\begin{array}{l}4.69 \\
(.73)\end{array}$ & $\begin{array}{l}4.69 \\
(.73) \\
\end{array}$ & $\begin{array}{l}4.62 \\
(.73)\end{array}$ & -.74 \\
\hline
\end{tabular}

Quadro 4. Problemas identificados na utilização de provas psicológicas

\begin{tabular}{|c|c|c|c|c|c|}
\hline & \multicolumn{2}{|c|}{ Amostra total } & \multirow{2}{*}{$\begin{array}{c}\text { Utilizadores } \\
M \\
(D P)\end{array}$} & \multirow{2}{*}{$\begin{array}{c}\text { Não-utilizadores } \\
M \\
(D P)\end{array}$} & \multirow[b]{2}{*}{$t$} \\
\hline & Mo & $\begin{array}{c}M \\
(D P)\end{array}$ & & & \\
\hline Fazer fotocópias dos testes & 5 & $\begin{array}{c}3.72 \\
(1.27)\end{array}$ & $\begin{array}{c}3.70 \\
(1.28)\end{array}$ & $\begin{array}{c}3.97 \\
(1.12)\end{array}$ & 1.89 \\
\hline Avaliar com testes inadequados & 3 & $\begin{array}{c}2.86 \\
(1.25)\end{array}$ & $\begin{array}{c}2.83 \\
(1.25)\end{array}$ & $\begin{array}{c}3.36 \\
(1.18)\end{array}$ & $3.46^{* *}$ \\
\hline Não estar atualizado(a) nos testes & 3 & $\begin{array}{c}3.18 \\
(1.19)\end{array}$ & $\begin{array}{c}3.14 \\
(1.20)\end{array}$ & $\begin{array}{l}3.72 \\
(.92)\end{array}$ & $5.00 * *$ \\
\hline Não contrastar avaliações com colegas & 4 & $\begin{array}{c}3.44 \\
(1.16)\end{array}$ & $\begin{array}{c}3.43 \\
(1.17)\end{array}$ & $\begin{array}{c}3.64 \\
(1.06)\end{array}$ & 1.44 \\
\hline Não ponderar erro padrão de medida & 3 & $\begin{array}{c}2.88 \\
(1.16)\end{array}$ & $\begin{array}{c}2.88 \\
(1.17)\end{array}$ & $\begin{array}{l}2.99 \\
(.99)\end{array}$ & .87 \\
\hline Uso por pessoas não qualificadas & 4 & $\begin{array}{c}3.17 \\
(1.43)\end{array}$ & $\begin{array}{c}3.14 \\
(1.44)\end{array}$ & $\begin{array}{c}3.54 \\
(1.28)\end{array}$ & $2.47 *$ \\
\hline Não considerar variáveis regionais/culturais & 3 & $\begin{array}{c}3.15 \\
(1.20)\end{array}$ & $\begin{array}{c}3.13 \\
(1.21)\end{array}$ & $\begin{array}{c}3.45 \\
(1.08)\end{array}$ & $2.16^{*}$ \\
\hline Interpretações sem ponderar limites do teste & 3 & $\begin{array}{c}3.13 \\
(1.20)\end{array}$ & $\begin{array}{c}3.11 \\
(1.20)\end{array}$ & $\begin{array}{c}3.48 \\
(1.08)\end{array}$ & $2.48^{*}$ \\
\hline
\end{tabular}

relativamente à satisfação com os testes, considerando os primeiros que estes lhes dão confiança e que são de grande ajuda na sua prática profissional.

As respostas às questões sobre a necessidade de regulamentação do uso de provas psicológicas (Quadro 2) sublinham uma atitude claramente favorável à necessidade de legislação e existência de normas nesta área, assinalando a importância da certificação de competências por parte dos utilizadores e o papel decisivo das associações e organismos profissionais nesta matéria. Relativamente a estas questões não se observam diferenças entre os respondentes que utilizam e os que não utilizam provas psicológicas.

No Quadro 3 registam-se as atitudes dos respondentes face ao uso exclusivo de provas psicológicas por parte dos psicólogos. Todas estas questões suscitaram uma adesão clara à ideia de que o uso de testes deve ser uma atividade da exclusiva responsabilidade dos psicólogos, no que diz respeito à administração, interpretação dos resultados e à sua comunicação. Também quanto a estas questões foi registado acordo entre os psicólogos que usam e os que não usam instrumentos de avaliação na sua prática.

O Quadro 4 permite identificar como problemas mais importantes para os respondentes, na utilização de provas psicológicas, o fazer fotocópias dos testes, seguido pelo não contrastar os resultados obtidos nas avaliações com outros colegas. Quando comparados com os psicólogos que utilizam instrumentos de avaliação na sua prática profissional, os que dizem não utilizar associam a esta prática vários tipos de problemas com mais intensidade, problemas estes relacionados, quer com a qualidade dos instrumentos (inadequados, sem considerar variáveis regionais/culturais), quer com a competência dos utilizadores (não atualizados quanto aos testes e sem ponderar os respetivos limites). Nestas situações, as diferenças entre as 
respostas dos dois grupos de respondentes assumem significância estatística.

\section{Áreas de atividade e de especialização}

Considerando os respondentes que realizam de forma habitual a avaliação psicológica na sua prática profissional $(n=1383)$, no Quadro 5 indicamos a distribuição dos respondentes pelas diferentes áreas de atividade profissional. Esta questão permite que o respondente, quando não exerce a sua atividade numa área em exclusivo, indique até três áreas de atividade profissional, por ordem de importância.

Quadro 5. Frequência de respostas para áreas de atividade profissional admitindo três

possibilidades de resposta

\begin{tabular}{|c|c|c|c|c|c|c|}
\hline \multirow{2}{*}{$\begin{array}{l}\text { Área de atividade } \\
\text { profissional }\end{array}$} & \multicolumn{2}{|c|}{$1^{\mathrm{a}}$ Opção } & \multicolumn{2}{|c|}{$2^{\mathrm{a}}$ Opção } & \multicolumn{2}{|c|}{$3^{\text {a }}$ Opção } \\
\hline & $n$ & $\%$ & $n$ & $\%$ & $n$ & $\%$ \\
\hline $\begin{array}{l}\text { Ensino Superior e } \\
\text { Investigação }\end{array}$ & 69 & 5.0 & 46 & 5.3 & 33 & 9.2 \\
\hline Neuropsicologia & 61 & 4.4 & 96 & 11.0 & 44 & 12.3 \\
\hline $\begin{array}{l}\text { Psicologia Clínica } \\
\text { e da Saúde }\end{array}$ & 676 & 48.9 & 283 & 32.5 & 85 & 23.8 \\
\hline Psicologia & & & & & & \\
\hline $\begin{array}{l}\text { Atividade Física } \\
\text { Desporto }\end{array}$ & 2 & .1 & 5 & .6 & 6 & 1.7 \\
\hline $\begin{array}{l}\text { Psicologia do } \\
\text { Trabalho } \\
\text { Organizacões }\end{array}$ & 78 & 5.6 & 57 & 6.5 & 27 & 7.6 \\
\hline $\begin{array}{l}\text { Psicologia Escolar } \\
\text { e da Educação }\end{array}$ & 377 & 27.3 & 222 & 25.5 & 56 & 15.7 \\
\hline $\begin{array}{l}\text { Psicologia Forense } \\
\text { e da Justiça }\end{array}$ & 38 & 2.7 & 44 & 5.1 & 30 & 8.4 \\
\hline $\begin{array}{l}\text { Psicologia Social e } \\
\text { Comunitária }\end{array}$ & 63 & 4.6 & 91 & 10.4 & 60 & 16.8 \\
\hline Outra & 19 & 1.4 & 27 & 3.1 & 16 & 4.5 \\
\hline TOTAL & 1383 & 100 & 871 & 100 & 357 & 100 \\
\hline
\end{tabular}

As áreas mais clássicas da prática profissional da Psicologia estão mais representadas na amostra, coincidindo, igualmente, com os três colégios de especialidade considerados pela OPP (Psicologia Clínica e da Saúde, Psicologia da Educação e Psicologia Social, do Trabalho e das Organizações). Esta situação está patente quando consideramos a "1a opção" dos respondentes, mantendo-se essa tendência nas opções seguintes, embora de forma mais diluída. Assim, os dados sugerem que quase $50 \%$ dos respondentes identificam a sua atividade profissional maioritariamente com a Psicologia Clínica e da Saúde, havendo também $27 \%$ que aponta a Psicologia Escolar e da Educação e cerca de 6\% que refere como atividade profissional dominante a Psicologia do Trabalho e das Organizações. Estas percentagens são próximas das disponibilizadas pela OPP (dados não publicados, tendo como referência o final do ano de 2017). Considerando ainda a $1^{\mathrm{a}}$ opção, cerca de $5 \%$ do total da amostra aponta o Ensino Superior e Investigação ou a Psicologia Social e Comunitária como áreas de atuação. De referir, ainda, apenas 3\% de respondentes na Psicologia Forense e da Justiça. Comparativamente com a $1^{a}$ opção, na $2^{\mathrm{a}}$ e na $3^{\mathrm{a}}$ opções são de destacar percentagens superiores a 10\% de psicólogos ligados à Neuropsicologia e Psicologia Social e Comunitária. Na categoria "Outra" predominam respostas como "Psicoterapia", "Necessidades Educativas Especiais" e "Psicogerontologia".

Por sua vez, no Quadro 6 apresentamos a área de formação especializada/pós-graduada dos respondentes. Também aqui eram facultadas três possibilidades de resposta, ordenadas de acordo com a sua importância da $1^{\mathrm{a}}$ até à $3^{\mathrm{a}}$ possibilidade, sendo a lista das áreas fornecida pelo próprio questionário. As áreas de especialização descritas correspondem muito de perto às áreas de atuação profissional, ou seja, predominam a Psicologia Clínica e da Saúde e a Psicologia Escolar e da Educação, havendo, neste caso, a salientar a formação em Neuropsicologia, que representa cerca de $7 \%$ dos respondentes. De novo, na categoria "Outra" aparecem referenciadas a "Psicoterapia", "Necessidades Educativas Especiais" e "Psicogerontologia".

Quadro 6. Área de especialização dos respondentes admitindo três possibilidades de resposta

\begin{tabular}{lcccccc}
\hline $\begin{array}{l}\text { Área de } \\
\text { especialização }\end{array}$ & \multicolumn{2}{c}{$1^{\text {a }}$ Opção } & \multicolumn{2}{c}{$2^{\text {a }}$ Opção } & \multicolumn{2}{c}{$3^{\text {a }}$ Opção } \\
\cline { 2 - 7 } & $n$ & $\%$ & $n$ & $\%$ & $n$ & $\%$ \\
\hline $\begin{array}{l}\text { Ensino Superior e } \\
\text { Investigação }\end{array}$ & 35 & 2.5 & 41 & 5.9 & 18 & 7.3 \\
$\begin{array}{l}\text { Neuropsicologia } \\
\text { Psicologia Clínica }\end{array}$ & 93 & 6.7 & 108 & 15.5 & 27 & 11.0 \\
e da Saúde & 716 & 51.8 & 210 & 30.2 & 46 & 18.7 \\
$\begin{array}{l}\text { Psicologia } \\
\text { Atividade Física }\end{array}$ & 4 & .3 & 6 & .9 & 3 & 1.2 \\
$\begin{array}{l}\text { Desporto } \\
\text { Psicologia do }\end{array}$ & & & & & & \\
Trabalho & 74 & 5.4 & 45 & 6.5 & 20 & 8.1 \\
$\begin{array}{l}\text { Organizações } \\
\text { Psicologia Escolar }\end{array}$ & 325 & 23.5 & 130 & 18.7 & 38 & 15.4 \\
e da Educação & & & & & & \\
Psicologia & 49 & 3.5 & 42 & 6.0 & 28 & 11.4 \\
$\begin{array}{l}\text { Forense e da } \\
\text { Justiça }\end{array}$ & & & & & & \\
Psicologia Social & 24 & 1.7 & 57 & 8.2 & 43 & 17.5 \\
e Comunitária & 63 & 4.6 & 56 & 8.1 & 23 & 9.3 \\
$\begin{array}{l}\text { Outra } \\
\text { TOTAL }\end{array}$ & 1383 & 100 & 695 & 100 & 246 & 99.9 \\
\hline
\end{tabular}


Quadro 7. Média (DP) das percentagens para cada objetivo de utilização, para a amostra total e comparação entre áreas

\begin{tabular}{|c|c|c|c|c|c|c|}
\hline & Amostra total & Clínica & Educação & $\begin{array}{c}\text { Trabalho \& } \\
\text { Org. }\end{array}$ & $F(2,1082)$ & $\begin{array}{c}\text { Comparações } \\
\text { múltiplas }\end{array}$ \\
\hline Rastreio & $\begin{array}{c}16.81 \\
(17.85)\end{array}$ & $\begin{array}{c}19.46 \\
(18.96)\end{array}$ & $\begin{array}{c}14.83 \\
(13.94)\end{array}$ & $\begin{array}{c}3.84 \\
(9.40)\end{array}$ & $32.89 * *$ & $\mathrm{C}>\mathrm{E}>\mathrm{TO}$ \\
\hline Avaliação/diagnóstico & $\begin{array}{c}49.67 \\
(26.91)\end{array}$ & $\begin{array}{c}56.79 \\
(25.68)\end{array}$ & $\begin{array}{c}47.32 \\
(21.38)\end{array}$ & $\begin{array}{c}22.50 \\
(27.74)\end{array}$ & $73.65 * *$ & $\mathrm{C}>\mathrm{E}>\mathrm{TO}$ \\
\hline Seleção & $\begin{array}{c}6.01 \\
(18.71)\end{array}$ & $\begin{array}{l}2.69 \\
(9.20)\end{array}$ & $\begin{array}{l}2.21 \\
(9.11)\end{array}$ & $\begin{array}{c}58.14 \\
(37.98)\end{array}$ & $613.52 * *$ & $\mathrm{TO}>\mathrm{C}, \mathrm{E}$ \\
\hline Orientação & $\begin{array}{c}12.73 \\
(17.61)\end{array}$ & $\begin{array}{c}8.02 \\
(12.29)\end{array}$ & $\begin{array}{c}26.49 \\
(21.53)\end{array}$ & $\begin{array}{c}6.30 \\
(11.17)\end{array}$ & $165.86 * *$ & $\mathrm{E}>\mathrm{C}, \mathrm{TO}$ \\
\hline Avaliação programas & $\begin{array}{c}6.81 \\
(11.89)\end{array}$ & $\begin{array}{c}6.84 \\
(13.17)\end{array}$ & $\begin{array}{c}6.29 \\
(9.00)\end{array}$ & $\begin{array}{c}3.99 \\
(9.59)\end{array}$ & 2.09 & \\
\hline Investigação & $\begin{array}{c}6.57 \\
(17.40)\end{array}$ & $\begin{array}{c}4.94 \\
(13.84)\end{array}$ & $\begin{array}{c}2.16 \\
(9.06)\end{array}$ & $\begin{array}{c}4.03 \\
(15.40)\end{array}$ & $5.71 *$ & $\mathrm{C}>\mathrm{E}$ \\
\hline Docência & $\begin{array}{c}1.14 \\
(5.51)\end{array}$ & $\begin{array}{c}.88 \\
(5.03)\end{array}$ & $\begin{array}{c}.55 \\
(3.37)\end{array}$ & $\begin{array}{c}1.20 \\
(5.22)\end{array}$ & .91 & \\
\hline Outro & $\begin{array}{c}.26 \\
(3.23) \\
\end{array}$ & $\begin{array}{c}.37 \\
(4.39) \\
\end{array}$ & $\begin{array}{c}.14 \\
(1.04)\end{array}$ & $\begin{array}{c}.00 \\
(.00)\end{array}$ & .77 & \\
\hline
\end{tabular}

Nota. As comparações múltiplas foram efetuadas com recurso ao Teste de Bonferroni. C=Psicologia Clínica e da Saúde; $\mathrm{E}=$ Psicologia Escolar e da Educação; TO=Psicologia do Trabalho e das Organizações.

$* p<.01 * * p<.001$

\section{Objetivos de utilização de provas psicológicas e idade da população-alvo}

No Quadro 7 são apresentadas as médias das percentagens de uso indicadas pelos respondentes para cada objetivo de utilização de instrumentos de avaliação psicológica. Nesta análise e na seguinte (Quadro 8) foram consideradas somente áreas de atuação que representam uma percentagem da amostra superior a $5 \%$ na Opção 1 (cf. Quadro 5), áreas estas que correspondem às especialidades definidas pela Ordem dos Psicólogos Portugueses. Tendo em conta o modo como está formulada esta questão (em que é pedido para indicar a percentagem de uso associada a cada objetivo), para esta análise foram eliminados todos os protocolos em que a soma das percentagens assinaladas para os diferentes objetivos era diferente de $100(n=25)$.

Tal como se pode observar, o objetivo associado a uma percentagem de utilização mais elevada é o de avaliação/diagnóstico (cerca de 50\%), seguido do rastreio (cerca de 17\%). A comparação entre profissionais das três áreas consideradas traduz-se em diferenças estatisticamente significativas para todos os objetivos de utilização, com exceção da avaliação de programas $(p=.125)$ e docência $(p=.404)$. As áreas diferem entre si quanto à utilização de instrumentos com objetivos de rastreio e avaliação/diagnóstico, sendo os psicólogos da área de Psicologia Clínica e da Saúde quem mais os utiliza com estes objetivos, seguidos pelos psicólogos ligados à Escola e Educação e, finalmente, ao Trabalho e Organizações. Compreensivelmente, o objetivo de seleção surge significativamente mais representado na área de Trabalho e Organizações, enquanto o de orientação está significativamente mais presente na área da Psicologia Escolar e da Educação. O objetivo de investigação está significativamente mais presente na área de Psicologia Clínica e da Saúde do que na de Psicologia Escolar e da Educação.

No Quadro 8 são apresentadas as médias das percentagens de uso de instrumentos de avaliação psicológica junto de destinatários de diferentes idades, sendo os intervalos etários decorrentes do esforço de conciliação das realidades préescolares, escolares e profissionais presentes nos vários países participantes no estudo. Tal como se pode verificar, em termos gerais os grupos etários dos 8 aos 12 anos, dos 13 aos 25 anos e os adultos até aos 64 anos são aqueles a que se associam maiores percentagens de uso destes instrumentos, representando, no conjunto, mais de $75 \%$ das utilizações. As faixas etárias extremas encontramse pouco representadas, com percentagens médias próximas de cerca de $8 \%$ para os adultos idosos, e de cerca de $2 \%$ para as crianças com idades até aos 3 anos.

Comparando psicólogos de áreas diferentes quanto à idade da população a quem aplicam instrumentos de avaliação psicológica (considerando nesta comparação somente as áreas 
Quadro 8. Média (DP) das percentagens para cada faixa etária da população destinatária para a amostra total e comparação entre áreas

\begin{tabular}{|c|c|c|c|c|c|c|}
\hline & Amostra Total & Clínica & Educação & $\begin{array}{l}\text { Trabalho \& } \\
\text { Org. }\end{array}$ & $F(2,1040)$ & $\begin{array}{c}\text { Comparações } \\
\text { múltiplas }\end{array}$ \\
\hline 0 a 3 anos & $\begin{array}{c}2.43 \\
(7.28)\end{array}$ & $\begin{array}{c}2.27 \\
(5.87)\end{array}$ & $\begin{array}{c}2.77 \\
(7.24)\end{array}$ & $\begin{array}{c}.74 \\
(3.85)\end{array}$ & $3.29 *$ & $\mathrm{E}>\mathrm{TO}$ \\
\hline 3 a 7 anos & $\begin{array}{c}13.50 \\
(16.35)\end{array}$ & $\begin{array}{l}13.20 \\
(15.95)\end{array}$ & $\begin{array}{c}18.82 \\
(16.90)\end{array}$ & $\begin{array}{c}4.73 \\
(11.41)\end{array}$ & $28.76 * *$ & $\mathrm{E}>\mathrm{C}>\mathrm{TO}$ \\
\hline 8 a 12 anos & $\begin{array}{c}24.04 \\
(22.33)\end{array}$ & $\begin{array}{c}23.51 \\
(22.23)\end{array}$ & $\begin{array}{c}34.92 \\
(20.68)\end{array}$ & $\begin{array}{c}8.38 \\
(16.62)\end{array}$ & $60.31 * *$ & $\mathrm{E}>\mathrm{C}>\mathrm{TO}$ \\
\hline 13 a 25 anos & $\begin{array}{l}28.26 \\
(22.87)\end{array}$ & $\begin{array}{l}24.76 \\
(20.04)\end{array}$ & $\begin{array}{c}37.61 \\
(25.61)\end{array}$ & $\begin{array}{c}23.15 \\
(19.86)\end{array}$ & $40.87 * *$ & $\mathrm{E}>\mathrm{C}, \mathrm{TO}$ \\
\hline Adultos até 64 & $\begin{array}{c}23.85 \\
(29.17)\end{array}$ & $\begin{array}{l}26.65 \\
(28.37)\end{array}$ & $\begin{array}{c}5.01 \\
(12.92)\end{array}$ & $\begin{array}{c}61.66 \\
(34.12)\end{array}$ & $189.85^{* *}$ & $\mathrm{TO}>\mathrm{C}>\mathrm{E}$ \\
\hline Adultos mais 64 & $\begin{array}{c}7.92 \\
(17.50)\end{array}$ & $\begin{array}{c}9.61 \\
(18.13)\end{array}$ & $\begin{array}{c}.87 \\
(4.28)\end{array}$ & $\begin{array}{l}1.34 \\
(3.48)\end{array}$ & $47.28 * *$ & $\mathrm{C}>\mathrm{E}, \mathrm{TO}$ \\
\hline
\end{tabular}

Nota. As comparações múltiplas foram efetuadas com recurso ao Teste de Bonferroni. C=Psicologia Clínica e da Saúde; E=Psicologia Escolar e da Educação; TO=Psicologia do Trabalho e das Organizações. $* p<.01 * * p<.001$

a que se associam mais de $5 \%$ dos utilizadores), verifica-se que eles diferem entre si em todas as faixas etárias. Assim, os psicólogos ligados à área Escolar/Educação são quem mais usa instrumentos de avaliação psicológica com crianças de idades compreendidas entre os 0 e os 3 anos (diferença estatisticamente significativa somente com Trabalho/Organizações). São também os psicólogos da área Escolar/Educação quem mais recorre a este tipo de instrumentos para avaliar crianças com idades entre os 3 e os 7 anos e entre os 8 e os 12 anos, seguidos pelos psicólogos da área de Psicologia Clínica/Saúde e, finalmente, de Trabalho/Organizações, sendo todas as diferenças estatisticamente significativas. A população dos 13 aos 25 anos de idade é significativamente mais avaliada com testes e questionários por psicólogos da área da Psicologia Escolar/Educação, em comparação com as outras duas áreas. A utilização de instrumentos com adultos até aos 64 anos é preferencialmente conduzida por psicólogos ligados à área de Trabalho/Organizações, seguidos pelos que exercem a sua atividade na Clínica/Saúde e, finalmente, na área de Psicologia Escolar/Educação (diferenças estatisticamente significativas entre todas as áreas). Os adultos com mais de 65 anos são avaliados com testes e questionários sobretudo por psicólogos ligados à área de Clínica/Saúde, sendo as diferenças estatisticamente significativas entre esta e as outras duas áreas.

\section{Discussão}

O presente estudo apoia-se numa amostra considerável de psicólogos portugueses, comparativamente a inquéritos anteriores (Almeida
\& Cruz, 1985; Diniz et al., 2007). Tal facto terá sido facilitado pela colaboração da OPP e pela natureza online do preenchimento do questionário. A generalidade dos psicólogos portugueses que participaram no estudo (em torno de 90\%) utiliza habitualmente testes ou questionários psicológicos na sua prática. Esta utilização generalizada atesta a relevância da avaliação psicológica na prática destes profissionais (Bernstein, 2017; Muñiz et al., 2011). Aliás, os que apontam que não utilizam, justificam-no por não ser necessário ou não fazer sentido dada a especificidade das suas funções (ação social, investigação, docência, direção/coordenação de serviços de psicologia, prática clínica, psicoterapia). Os utilizadores são em maior proporção do sexo feminino e psicólogos mais velhos ou com mais anos de prática. Estas diferenças podem estar relacionadas com as áreas de intervenção. Assim, por exemplo psicólogos mais jovens e em áreas mais recentes da prática psicológica, como seja a Psicologia Social-Comunitária, poderão recorrer menos a estas práticas. O contrário poderá ocorrer junto de psicólogos mais velhos e provavelmente mais do sexo feminino, que poderão exercer a sua atividade nas áreas clássicas, como a Psicologia Escolar e da Educação e a Psicologia Clínica e da Saúde, com maior tradição da prática de avaliação psicológica. Assim, em linha com outros estudos anteriores em Portugal, assiste-se a uma atitude geral de aceitação do método dos testes e sua utilização, como aliás acontece noutros países europeus (Cruz \& Almeida, 1985; Diniz et al., 2007; Muñiz et al., 2011).

A generalidade dos psicólogos portugueses que 
participaram no estudo, em linha com estudos anteriores em Portugal, expressa a sua satisfação com a qualidade dos testes que utiliza, assumindo que, quando usados de forma adequada, os testes são de grande ajuda e dão confiança à sua prática (Cruz \& Almeida, 1985; Diniz, Almeida, \& Pais, 2007; Muñiz et al., 2011). Importa destacar que os psicólogos que não recorrem na sua prática à utilização de testes psicológicos apresentam níveis mais baixos de acordo nos itens relativos à satisfação com a qualidade dos testes, com diferenças de pontuação estatisticamente significativas. Esta menor satisfação com a qualidade dos testes pode constituir tanto a causa como a consequência de uma menor utilização destes instrumentos, na sua prática profissional. No entanto, poderá traduzir, também, atitudes mais críticas em relação ao próprio método dos testes, quer no que diz respeito à sua relevância, quer nas suas caraterísticas métricas. No sentido da relevância da formação na área, a generalidade da amostra expressa o seu acordo quanto à necessidade de unidades curriculares nos cursos de Psicologia sobre construção e avaliação de testes, sendo que já num estudo anterior os psicólogos portugueses apontavam também a necessidade de formação contínua ou pósgraduada na área da avaliação (Diniz et al., 2007).

Os inquiridos manifestam acordo quanto à necessidade de regulamentação do uso das provas psicológicas, sendo esta atitude igualmente assumida pelos psicólogos que utilizam e que não utilizam as mesmas provas na sua prática profissional. Esta atitude consensual de maior regulação e da intervenção ou papel mais ativo das associações de psicologia/psicólogos nessa regulação é comum a outros estudos e países (Muñiz et al., 1999). A defesa desta regulamentação, mesmo por parte de quem não utiliza as provas psicológicas, sugere estarmos face a uma questão de identidade e de maturidade da prática de avaliação psicológica. Este ponto sai reforçado quando se questionam os psicólogos sobre a exclusividade da sua atuação em vários momentos da avaliação psicológica e do uso dos testes, havendo um claro assumir que as decisões a tomar neste contexto são da competência específica dos psicólogos, sendo esta posição consensual e não se registando também aqui diferenças nas pontuações de psicólogos que usam ou não usam habitualmente provas psicológicas na sua prática. A utilização dos testes psicológicos é encarada como sendo da exclusiva responsabilidade dos psicólogos, incluindo aqui a sua administração, interpretação e comunicação dos resultados. Estes dados vão no mesmo sentido dos obtidos no estudo Ricou, Cordeiro, Franco e Costa-Lobo (2018), no qual a avaliação psicológica surge como a atividade mais consensualmente encarada, pelos psicólogos participantes no estudo, como função exclusiva de psicólogos. Neste contexto, podemos pensar que a marca identitária que a avaliação psicológica constitui para os psicólogos portugueses terá sido reforçada pela criação da Ordem (OPP), cujo papel regulador constituiu uma novidade para a prática da Psicologia em Portugal ao longo da última década. Neste contexto é de realçar a publicação do Código Deontológico da OPP (2011) com uma secção direcionada para os princípios específicos da avaliação psicológica e, mais recentemente, a criação do Conselho Consultivo para a Acreditação de Testes e Provas Psicológicas da OPP (2018).

Quanto aos problemas identificados na utilização das provas psicológicas, a generalidade dos psicólogos expressa uma preocupação moderada relativamente aos vários pontos elencados, sendo a utilização de provas fotocopiadas o problema mais valorizado. A ausência de interação com outros colegas para apreciar os resultados obtidos nas avaliações, o não ponderar os limites dos próprios testes na interpretação dos resultados, o uso de provas sem a suficiente qualificação ou a insuficiente atualização nos testes que utiliza, ou mesmo o uso de testes não adequados, são problemas moderadamente identificados na nossa amostra. De destacar, igualmente, a preocupação com a necessidade de os testes e outros procedimentos de avaliação psicológica atenderem a variáveis regionais e culturais com impacto no desempenho dos avaliados, condição essencial da própria validade da avaliação psicológica (Casullo, 2009).

Este conjunto de preocupações foi mais assinalado por psicólogos que mencionam não usar as provas psicológicas habitualmente na sua prática. Tal diferença, assumindo significância estatística, sugere um olhar particularmente crítico por parte deste subgrupo de psicólogos, que pode 
estar na origem da não utilização das provas. É possível, também, que a não utilização das provas proporcione um distanciamento face às mesmas que justifique este maior sentido crítico. Trata-se de problemas que emergem em estudos similares, em Portugal e noutros países, expressando a sensibilidade técnica e social do uso das provas psicológicas (Almeida, Araújo, \& Diniz, 2013; Diniz et al., 2007; Muñiz et al., 1999; Pallás \& Llorens, 2010). Assim, é de salientar que os psicólogos portugueses parecem ter assimilado princípios fundamentais da avaliação psicológica em geral e do uso de testes em particular, tal como estão consignados em diretrizes internacionais (American Educational Research Association, American Psychological Association, American Council on Measurement in Education, 2014; International Test Commission, 2013, 2018) e nacionais (Ordem dos Psicólogos Portugueses, 2011).

Finalmente, questionados quanto ao objetivo da administração de testes, uma maior percentagem de respondentes aponta para uma utilização voltada para a avaliação/diagnóstico, perfazendo, com o objetivo de rastreio, um total de $67 \%$ das utilizações. Estes objetivos estão particularmente presentes junto dos psicólogos da área de Psicologia Clínica e da Saúde, seguidos pelos psicólogos ligados à Escola e Educação. Como seria expectável, o objetivo de seleção surge significativamente mais representado na área de Trabalho e Organizações, e o objetivo da orientação mais presente na área da Psicologia Escolar e da Educação. O objetivo de investigação está significativamente mais presente na área de Psicologia Clínica e da Saúde do que na de Psicologia Escolar e da Educação, o que poderá estar relacionado com um maior número de oportunidades de formação em serviço por parte dos primeiros ou, ainda, com o enquadramento em equipas multidisciplinares que incluem outros profissionais orientados para a investigação, nomeadamente médicos.

Em termos do público-alvo, os grupos etários dos 8 aos 12 anos, dos 13 aos 25 anos e dos adultos até aos 64 anos representam mais de $75 \%$ das utilizações das provas psicológicas. As faixas etárias extremas encontram-se pouco representadas, podendo não refletir mudanças recentes do desenvolvimento da investigação com provas psicológicas. Nomeadamente, os adultos idosos constituem a faixa etária na qual mais cresceu a investigação com testes, na última década, em Portugal (Simões, 2012), o que é evidenciado pelos 32 instrumentos de avaliação com estudos de validação recentes na população idosa portuguesa, recenseados no livro de Simões e Santana (2015).

Este estudo comporta algumas limitações, que importa assinalar. A tentativa de encontrar uma formulação comum do questionário para poder ser utilizado em diferentes países que participam no estudo internacional pode ter criado, eventualmente, dificuldades de compreensão em algumas questões (e.g., alternativas de resposta facultadas para as perguntas sobre a formação académica, áreas de especialização e de intervenção, objetivos da aplicação de testes). Por outro lado, o facto de ser preenchido online, ao mesmo tempo que permitiu obter respostas de um número expressivo de psicólogos, pode ter limitado o acesso a profissionais menos familiarizados com as tecnologias digitais.

Os resultados obtidos com a realização deste inquérito permitem compreender o perfil dos utilizadores de instrumentos de avaliação psicológica em Portugal, as suas atitudes em relação a esses instrumentos e os problemas que os preocupam nesta área. Estudos futuros poderão comparar os resultados portugueses com os obtidos noutros países latino-americanos que participaram no estudo mais alargado, permitindo perspetivar estes dados numa escala internacional.

\section{Referências}

Almeida, L. S., \& Cruz, O. (1985). A utilização dos testes psicológicos: Resultados de um inquérito junto de psicólogos portugueses. Jornal de Psicologia, 4(5), 11-18.

Almeida, L. S., Araújo, A. M., \& Diniz, A. M. (2013). Avaliação psicológica e o uso dos testes em Portugal. Psiencia - Revista Latinoamericana de Ciencia Psicológica, $5(2)$, http://dx.doi.org/10.5872/psiencia.v5i2.119

American Educational Research Association, American Psychological Association, American Council on Measurement in 
Education (2014). Standards for educational and psychological testing ( $3^{\text {rd }}$ ed.). Washington, DC: American Educational Research Association.

Archer, R. P., Buffington-Vollum, J. K., Stredny, R. V., \& Handel, R. W. (2006). A survey of psychological test use patterns among forensic psychologists. Journal of Personality Assessment, $\quad 87(1), \quad 84-94$. https://doi.org/10.1207/s15327752jpa8701_07

Bornstein, R. F. (2017). Evidence-based psychological assessment. Journal of Personality Assessment, 99(4), 435-445. https://doi.org/10.1080/00223891.2016.12363 43

Camara, W. J., Nathan, J. S., \& Puente, A. E. (2000). Psychological test usage: Implications in professional psychology. Professional Psychology: Research and Practice, 31(2), 141-154.

http://dx.doi.org/10.1037/0735-7028.31.2.141

Casullo, M. M. (2009). La evaluación psicológica: Modelos, técnicas y contextos. Revista Iberoamericana de Diagnóstico y Evaluación - e Avaliação Psicológica, 27(1), 9-28. http://www.redalyc.org/articulo.oa?id=459645 443002

Cruz, O., \& Almeida, L. S. (1987). A utilização dos testes psicológicos em Portugal: Análise segundo a formação e o domínio de prática profissional. Jornal de Psicologia, 6(1), 1319.

Diniz, A. M., Almeida, L. S., \& Pais, L. G. (2007). Contextos profissionais e práticas de avaliação psicológica: Inquérito aos psicólogos portugueses. Psico-USF, 12(1), 1-12. http://dx.doi.org/10.1590/S141382712007000100002

International Test Commission. (2013). ITC Guidelines on Test Use. Acedido de https://www.intestcom.org/files/guideline_test _use.pdf

International Test Commission. (2018). The ITC guidelines for translating and adapting tests ( $2^{\text {nd }} \quad$ edition). Acedido de http://www.InTestCom.org

Muñiz, J., Bartram, D., Evers, A., Boben, D., Matesic, K., Glabeke, K., FernándezHermida, J. R., \& Zaal, J. N. (2011). Testing practices in european countries. European
Journal of Psychological Assessment, 17(3), 201-211.

https://doi.org/10.1027/1016-9040/a000102.

Muñiz, J., Prieto, G., Almeida, L. \& Bartram, D. (1999). Test use in Spain, Portugal and latin american countries. European Journal of Psychological Assessment, 15(2), 151-157. https://doi.org/10.1027//1015-5759.15.2.151

Ordem dos Psicólogos Portugueses. (2011). Código deontológico. Acedido de https://www.ordemdospsicologos.pt/pt/cod_de ontologico

Ordem dos Psicólogos Portugueses. (2018). Conselho Consultivo para a Acreditação de Testes e Provas Psicológicas. Acedido de https://www.ordemdospsicologos.pt/pt/comiss oes/comissao/index/conselho-consultivo-paraa-acreditacao-de-testes-e-provas-psicologicasda-opp

Pallás, C. M., \& Llorens, O. G. (2010). Enfoque de la evaluación psicológica de la Revista Iberoamericana de Diagnóstico y Evaluación Psicológica. Revista Iberoamericana de Diagnóstico y Evaluación - e Avaliação Psicológica, 30(2), 35-55. http://www.redalyc.org/articulo.oa?id=459645 442003

Ricou, M., Cordeiro, S. A., Franco, A. R., \& Costa-Lobo, C. (2018). The identity of psychology. The Psychologist: Practice and Research Journal, 1(1) 1-15. https://doi.org/10.33525/pprj.v1i1.25

Simões, M. R. (2012). Instrumentos de avaliação psicológica de pessoas idosas: Investigação e estudos de validação em Portugal. Revista Iberoamericana de Diagnóstico y Evaluación - e Avaliação Psicológica, 34(1), 9-33. http://www.aidep.org/03_ridep/R34/ART\%20 1.pdf

Simões, M. R., Santana, I., \& Grupo de Estudos de Envelhecimento Cerebral e Demência (Eds.) (2015). Escalas e testes na demência ( $3^{\mathrm{a}}$ edição). Porto Salvo: Novartis. 